\section{Criteria for early detection of temporal hemianopia in asymptomatic pituitary tumor}

N Fujimoto ${ }^{1}$, N Saeki ${ }^{2}$, O Miyauchi ${ }^{1}$

and $\mathrm{E}$ Adachi-Usami ${ }^{1}$

Abstract

Purpose To determine new criteria for early detection of temporal hemianopia in patients with asymptomatic pituitary tumor.

Methods Fifteen patients without visual symptoms had pituitary tumor and subtle defects or normal visual fields by Goldmann perimetry. Twelve patients with visual symptoms had pituitary adenoma and visual field defects detected by Goldmann perimetry. All were examined by automated perimetry. The relationship between the tumor and the optic chiasm was graded by magnetic resonance imaging (MRI) on a scale of 0-4. Grade number increased with extent of compression. Temporal hemianopia observed by automated perimetry was estimated from normal data (52 normal fields). Vertical step was determined from the number of adjacent pairs along the midline; sensitivity was lower in the temporal field than in the nasal field. Temporal depression was calculated by the quadrant sum of sensitivity.

Results All patients with symptomatic pituitary adenoma had vertical step and temporal depression in the upper field. Nine of 15 patients without visual symptoms had vertical step or temporal depression. Of nine patients with temporal hemianopia, two of four patients had grade 1 compression, three of five had grade 2, and four of four had grade 3.

Conclusion New criteria by automated perimetry could detect temporal hemianopia in patients with asymptomatic pituitary tumor.

Eye (2002) 16, 731-738. doi:10.1038/

sj.eye. 6700165

Keywords: asymptomatic; temporal hemianopia; vertical step; automated perimetry; pituitary tumor

A pituitary tumor can be located at the various positions of the optic chiasm. Visual field defects are produced by lesions at the anterior angle, body, posterior angle, or lateral aspect of the chiasm. A pituitary tumor frequently causes bitemporal hemianopia by compressing the optic chiasm initially. Visual field defects are thought to be due to the direct compression or the effect on the blood supply of the optic chiasm. These visual field defects can be detected by manual perimetry, ${ }^{1}$ and by automated static perimetry. ${ }^{2-5}$

Automated perimetry can find as many or more abnormalities in chiasmal lesions as can Goldmann perimetry. Automated static perimetry expresses numeric values as sensitivity and decreased values as depression. Vertical step or decreased value along the midline are known to be the initial findings of bitemporal hemianopia. ${ }^{5}$ However, these findings are not quantitatively determined. It is not yet known how many $\mathrm{dB}$ of differences in sensitivity between midlines could be significant. We also need to determine how many $\mathrm{dB}$ of decreased sensitivity in the temporal area lead to initial change. Magnetic resonance imaging (MRI) demonstrates compression to the optic chiasm by the tumor. Compression of the optic chiasm by the tumor can induce visual field loss. We studied the visual field by automated perimetry in patients with asymptomatic pituitary tumor demonstrated by MRI and determined the criteria of detecting temporal hemianopia.

\section{Patients and methods}

Fifteen patients (five men and 10 women) with pituitary tumor detected by MRI had no
'Department of

Ophthalmology and Visual Science

Chiba University Graduate School of Medicine

Chiba, Japan

${ }^{2}$ Dept of Neurological Surgery Chiba University Graduate School of Medicine

Chiba, Japan

Correspondence:

N Fujimoto, MD

Department of

Ophthalmology

Chiba University School of

Medicine

1-8-1 Inohana

Chuo-ku

Chiba 260, Japan

Tel: 00181432227171

ext 6186

Fax: 00181432244162

E-mail: fujimoto@

ophthalm.m.chiba-u.ac.jp

Received: 30 July 2001

Accepted: 8 March 2002 
visual complaints. Their ages ranged from 22 to 77 years (mean: 52.3 years). They had no ocular disease, including that of the optic nerve. Two patients had acromegaly and no compression to the optic chiasm, while 13 patients had pituitary tumor, including two prolactinomas touching or compressing the optic chiasm. Another 12 patients (four men and eight women) had pituitary adenoma and temporal hemianopia observed with Goldmann perimetry preoperatively. Their ages ranged from 13 to 59 years (mean: 39.5 years). These patients underwent surgical removal of the tumor. Four patients had hormoneproducing adenoma (one prolactin, two growth hormones, and one adrenocorticotrophic hormone), and eight had non-functioning adenoma. Fifty-two normal volunteers (26 men and 26 women) had neither ophthalmologic nor neurologic disease. Their ages ranged from 16 to 71 years (mean: 40.6 years).

The relationship between optic chiasm and tumor in 27 patients with tumor was estimated by coronal view on MRI, which was performed at $1.5 \mathrm{~T}$. Slice thickness was $3 \mathrm{~mm}$ with no interslice gap. T1-weighted images were obtained using TR/TE acquisitions of 600/20/2 The matrix was $256 \times 256$, and field of view was 20 $\mathrm{cm}$. The relationship was graded from 0 to 4 (Figure 1). One neurologist (NS) determined grade without knowing the visual field. In case of asymmetry of grade bilaterally, the higher grade was adopted. In case of posterior or anterior enlargement, the most prominent relationship was adopted. Grade 0 was defined as no contact between tumor and optic chiasm. Grade 1 indicated contact between them without upper surface deformity of optic chiasm. Grade 2

demonstrated contact, with upper surface deformity of optic chiasm and visible suprachiasmatic cistern. Grade 3 showed contact, with upper surface deformity and invisible suprachiasmatic cistern and no cerebral deformity. Grade 3+ meant MRI grade 3 with Goldmann visual field defects (Figures 2 and 3). Grade 4 indicated contact, with cerebral deformity. All patients were examined with use of the Humphrey field analyzer (Carl Zeiss, Dublin, CA, USA). The program used threshold central 30-2 (76 test points within 30 degree visual field). Results with more than $20 \%$ false positive, $20 \%$ false negative, or $20 \%$ fixation loss were excluded and reexamined. Some patients were reexamined within 6 months.

We set vertical step and temporal depression for the criteria of detecting temporal hemianopia to differentiate data of healthy subjects from those of patients with pituitary adenoma and temporal hemianopia observed by Goldmann perimetry.

The research followed institutional guidelines and the tenets of the World Medical Association

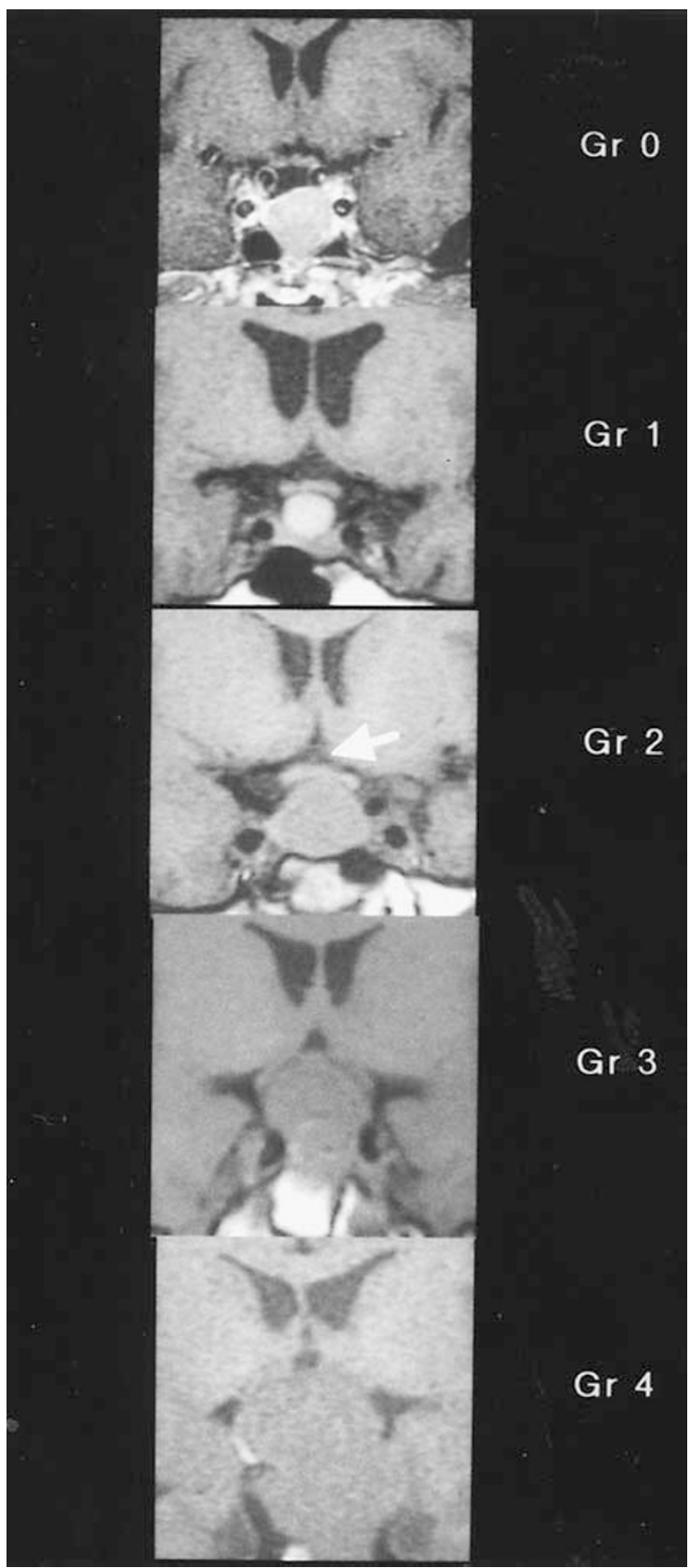

Figure 1 MRI compression grade was obtained from T1weighted coronal view. Grade $0(\mathrm{Gr} 0)$ tumor has no contact with optic chiasm. Grade 1 (Gr 1) tumor has contact with optic chiasm without the deformity of upper surface of optic chiasm. Grade 2 (Gr 2) tumor compressed optic chiasm and produced the deformity of the upper surface and visible suprachiasmal cistern (arrow head). Grade 3 (Gr 3) tumor compressed optic chiasm with invisible suprachiasmal cistern. Grade 4 (Gr 4) tumor compressed optic chiasm with cerebral deformity.

Declaration of Helsinki. We obtained informed consent from each subject studied. 


\section{Results}

Vertical step was determined from the results in healthy volunteers (Figure 2). There were maximally three continuous pairs, in whom each sensitivity was 2 $\mathrm{dB}$ or lower in the temporal field along the vertical meridian than in the corresponding nasal field in healthy subjects. There were maximally two adjacent pairs, in whom each sensitivity was $3 \mathrm{~dB}$ or lower in the temporal field along the vertical meridian than in the corresponding nasal field in healthy volunteers. Vertical temporal step was determined as significant when four or more adjacent pairs had $2 \mathrm{~dB}$ or more decreased sensitivity at the temporal midline, and when three or more adjacent pairs had $3 \mathrm{~dB}$ or more decreased sensitivity at the temporal midline. Temporal depression was obtained from printout 3 in 1 (gray scale, defects, and actual value). This printout showed the sum of sensitivity in each quadrant field in actual values. The difference in sensitivity between temporal and nasal quadrant fields (nasal-temporal) divided by sensitivity in the temporal quadrant field was determined as a temporal depression index (Figure 4). This index was equivalent to (nasal sensitivity /temporal sensitivity) -1 . Minus value meant nasal depression. Normal upper limit (mean +2 standard deviations) was 0.086 in upper pairs and 0.084 in lower pairs (Figure 3 ). One healthy person showed high temporal depression index (false positive).

All 12 symptomatic patients with pituitary adenoma had both $2 \mathrm{~dB}$ and $3 \mathrm{~dB}$ vertical temporal steps in either eye (Figure 2). Only one patient showed normal vertical steps in one eye (false negative). Mean $2 \mathrm{~dB}$ vertical step pairs were eight pairs. Mean $3 \mathrm{~dB}$ vertical step pairs were 7.8 pairs. All patients with pituitary adenoma had a significant temporal depression index

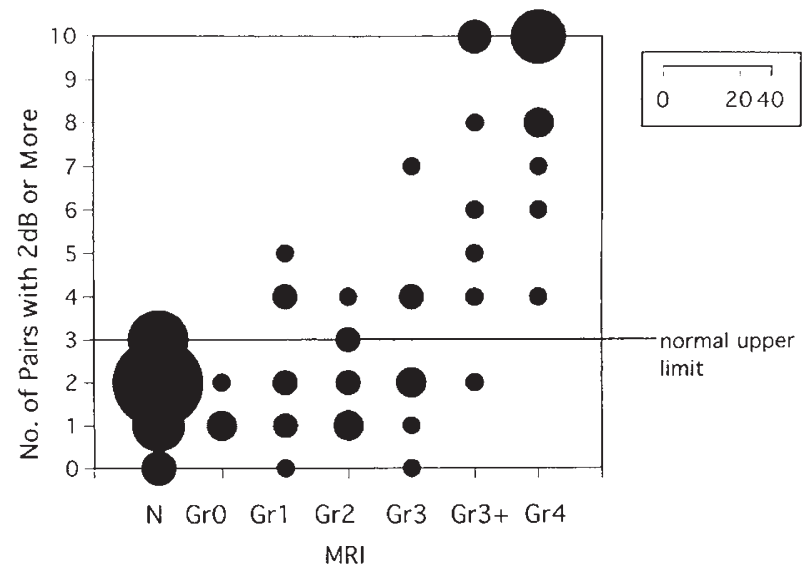

Figure 2 Number of adjacent pairs with $2 \mathrm{~dB}$ or lower sensitivity vs MRI grade is shown. $\mathrm{N}$ indicates normal healthy subjects. Gr $3+$ means MRI grade 3 with Goldmann visual field defects.

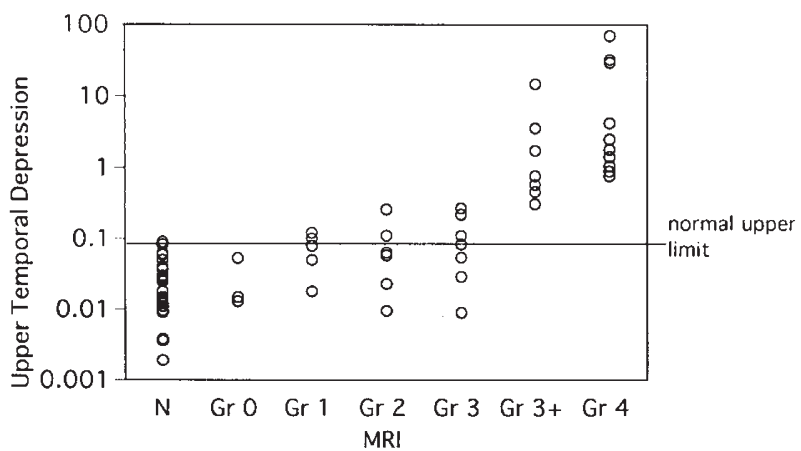

Figure 3 Upper temporal depression index vs MRI compression grade is shown. $\mathrm{N}$ indicates normal healthy subjects. Gr 3+ means MRI grade 3 with Goldmann visual field defects. Seven eyes with MRI grade 3+ or 4 had infinity in upper temporal depression index, which is not shown in Figure 3.

in the upper field (Figure 3). Eleven of 12 patients had a significant temporal depression in the lower field. From these findings, vertical step ( $2 \mathrm{~dB}$ or $3 \mathrm{~dB})$ and temporal depression (upper or lower) were determined as the criteria for detecting temporal hemianopia. Vertical step had $96 \%$ sensitivity and $100 \%$ specificity. Temporal depression had $100 \%$ sensitivity and $98 \%$ specificity. MRI demonstrated grade 3 (grade $3+$ in Figures 2 and 3) or grade 4 compression in all symptomatic patients.

Twenty-eight reliable visual fields were initially obtained from 15 patients with pituitary tumor without visual symptoms. One patient was reexamined immediately because of unreliable results and findings from the second examination were adopted. Five patients demonstrated abnormal visual field by Goldmann perimetry, but no hemianopic isopters (isopters that ran parallel to the vertical midline) and no scotoma. Five patients had a depression around the blind spot observed by Goldmann perimetry. Two patients with acromegaly and no compression to the optic chiasm showed no vertical temporal step and no temporal depression noted by automated perimetry. Thirteen of the other 26 visual fields had temporal hemianopia (Table 1). Seven of 13 patients with pituitary tumor had $2 \mathrm{~dB}$ vertical temporal step. Five had $3 \mathrm{~dB}$ vertical temporal step. Six had significant temporal depression in the upper field; one had depression in the lower field. Nine of 13 patients had vertical step or temporal depression. Four had vertical step or temporal depression in both eyes. Six had both vertical step and temporal depression. Eleven eyes of eight patients demonstrated abnormal field loss on automated perimetry, but normal field by Goldmann perimetry. Two patients had grade 0 , four had grade 1 , five had grade 2, and four had grade 3 compression on MRI findings. Grade 3 compression induced an 


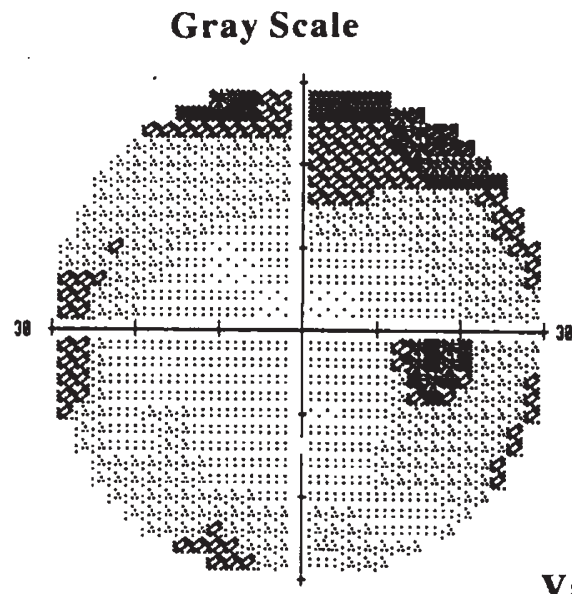

$\operatorname{Value}(\mathrm{dB})$
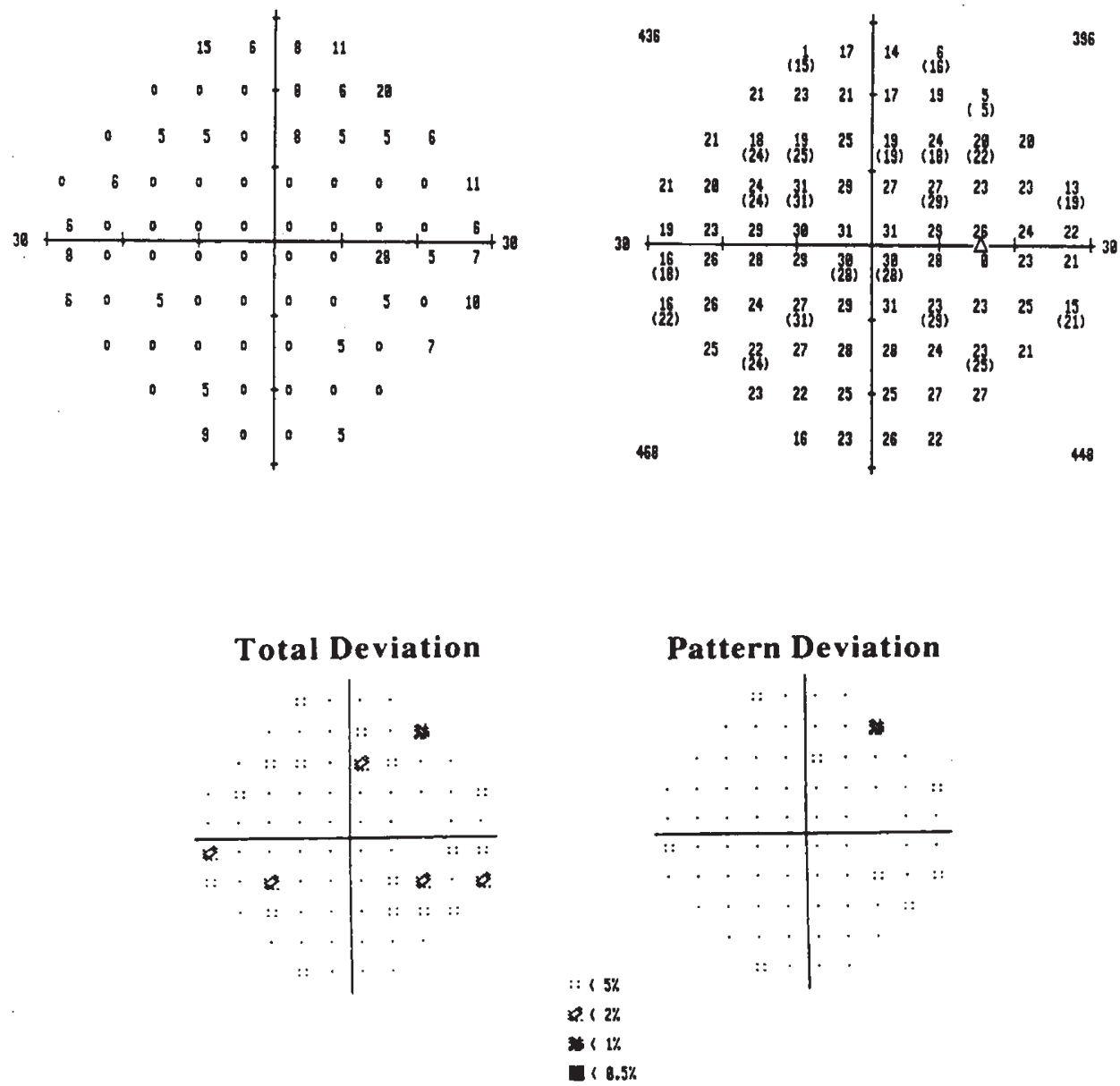

Figure 4 Temporal depression index is calculated by the quadrant sum obtained from the printout 3 in 1 . Temporal depression index $=$ (nasal quadrant sum - temporal quadrant sum $) /$ temporal quadrant sum. The 3 in 1 printout and deviation maps in right eye of patient 3 (65-year-old woman) are shown. Temporal depression index in upper field is (436-396)/396 = 0.10 in the right eye, which is higher than normal upper limit (0.086).

abnormal field loss by automated perimetry in all four patients. Grade 1 compression produced an abnormal field in two of four patients; Grade 2, three of five.

Eight of nine patients with temporal hemianopia determined by vertical step or temporal depression were reexamined within 6 months. Ten of 11 visual fields with temporal hemianopia were determined as temporal hemianopia again. One case (No. 8 in Table 
Table 1 Summary of asymptomatic pituitary tumor

\begin{tabular}{|c|c|c|c|c|c|c|c|c|c|c|}
\hline \multirow[t]{2}{*}{ Patient No. } & \multirow{2}{*}{$\begin{array}{c}\text { Age } \\
\text { (years) }\end{array}$} & \multirow[t]{2}{*}{ Sex } & \multicolumn{2}{|c|}{ Temporal depression } & \multicolumn{2}{|c|}{ No. of pairs } & \multirow{2}{*}{$\begin{array}{l}\text { Goldmann } \\
\text { perimeter }\end{array}$} & \multirow{2}{*}{$\begin{array}{l}\text { Visual } \\
\text { acuity }\end{array}$} & \multirow[t]{2}{*}{ Hormone } & \multirow{2}{*}{$\begin{array}{c}\text { MRI grade } \\
\text { tumor } \\
\text { compression }\end{array}$} \\
\hline & & & Upper & Lower & $2 d B$ or more & $3 d B$ or more & & & & \\
\hline \multirow[t]{2}{*}{1} & 41 & $\mathrm{~F}$ & 0.0130 & 0.0220 & 1 & 1 & $\mathrm{~N}$ & 1.5 & $\mathrm{GH}$ & 0 \\
\hline & & & -0.0660 & 0.0000 & 2 & 0 & $\mathrm{~N}$ & 1.2 & & 0 \\
\hline \multirow[t]{2}{*}{2} & 56 & M & 0.0530 & 0.0057 & 1 & 0 & $\mathrm{~N}$ & 1.2 & $\mathrm{GH}$ & 0 \\
\hline & & & 0.0150 & 0.0430 & 1 & 0 & $\mathrm{~N}$ & 1.5 & & 0 \\
\hline \multirow[t]{2}{*}{3} & 65 & $\mathrm{~F}$ & $\underline{0.1000}$ & 0.0450 & $\underline{4}$ & $\underline{3}$ & $\mathrm{~N}$ & 1.0 & $\mathrm{NF}$ & 1 \\
\hline & & & 0.0500 & 0.0230 & $\underline{5}$ & $\frac{-}{1}$ & $\mathrm{~N}$ & 1.0 & & 1 \\
\hline \multirow[t]{2}{*}{4} & 36 & $\mathrm{~F}$ & $\underline{0.1200}$ & $\underline{0.1340}$ & $\underline{\overline{4}}$ & $\underline{4}$ & $\mathrm{~N}$ & 1.2 & PRL & 1 \\
\hline & & & $\overline{0.0790}$ & $\overline{0.0630}$ & $\overline{2}$ & $\overline{2}$ & $\mathbf{A}$ & 1.2 & & 1 \\
\hline \multirow[t]{2}{*}{5} & 60 & F & -0.0018 & 0.0310 & 2 & 0 & $\mathrm{~N}$ & 1.2 & NF & 1 \\
\hline & & & -0.0940 & 0.0200 & 0 & 0 & $\mathrm{~N}$ & 1.0 & & 1 \\
\hline \multirow[t]{2}{*}{6} & 47 & F & 0.0180 & 0.0690 & 1 & 1 & $\mathrm{~N}$ & 1.0 & $\mathrm{NF}$ & 1 \\
\hline & & & -0.0850 & 0.0160 & 1 & 1 & $\mathrm{~N}$ & 1.2 & & 2 \\
\hline \multirow[t]{2}{*}{7} & 53 & F & 0.0970 & 0.0940 & $\underline{4}$ & 0 & $\mathrm{~N}$ & 1.0 & NF & 2 \\
\hline & & & 0.1090 & 0.2300 & $\frac{x}{2}$ & 0 & $\mathrm{~N}$ & 1.0 & & 2 \\
\hline \multirow[t]{2}{*}{8} & 58 & M & $\overline{0.1100}$ & $\overline{0.0830}$ & 3 & 2 & $\mathrm{~N}$ & 1.0 & NF & 2 \\
\hline & & & $\underline{0.2600}$ & $\underline{0.1500}$ & 3 & $\underline{3}$ & $\mathrm{~N}$ & 0.5 & & 2 \\
\hline \multirow[t]{2}{*}{9} & 77 & M & $\overline{0.0580}$ & 0.0640 & 2 & 0 & $\mathrm{~N}$ & 1.0 & NF & 2 \\
\hline & & & -0.0320 & 0.0039 & 1 & 1 & $\mathrm{~N}$ & 1.2 & & 2 \\
\hline \multirow[t]{2}{*}{10} & 26 & F & 0.0230 & 0.0250 & 1 & 0 & $\mathrm{~N}$ & 1.0 & NF & 2 \\
\hline & & & 0.0095 & 0.0370 & 2 & 0 & $\mathrm{~N}$ & 1.0 & & 2 \\
\hline \multirow[t]{2}{*}{11} & 22 & F & -0.0040 & 0.0140 & 1 & 1 & A & 1.5 & NF & 2 \\
\hline & & & 0.0640 & 0.0330 & $\underline{5}$ & 0 & $\mathrm{~N}$ & 1.2 & & 2 \\
\hline \multirow[t]{2}{*}{12} & 61 & M & 0.0290 & 0.0680 & $\overline{2}$ & 1 & $\mathbf{A}$ & 0.9 & $\mathrm{NF}$ & 3 \\
\hline & & & 0.0830 & 0.0770 & $\underline{4}$ & 3 & $\mathrm{~N}$ & 0.9 & & 3 \\
\hline \multirow[t]{2}{*}{13} & 65 & $\mathrm{~F}$ & $\underline{0.2700}$ & $\underline{0.1500}$ & $\underline{\overline{7}}$ & $\underline{5}$ & $\mathrm{~N}$ & 1.0 & NF & 3 \\
\hline & & & 0.0090 & $\overline{0.0530}$ & 2 & 2 & A & 1.0 & & 3 \\
\hline \multirow[t]{2}{*}{14} & 60 & $\mathrm{~F}$ & 0.1100 & -0.0048 & 2 & 1 & A & 1.0 & PRL & 3 \\
\hline & & & $\overline{0.2200}$ & -0.1200 & $\underline{4}$ & $\underline{3}$ & A & 1.2 & & 3 \\
\hline \multirow[t]{2}{*}{15} & 57 & M & $-\overline{0.0620}$ & -0.0980 & $\overline{1}$ & $\overline{0}$ & $\mathrm{~N}$ & 1.0 & NF & 3 \\
\hline & & & 0.0540 & $\underline{0.3100}$ & 0 & 0 & $\mathrm{~N}$ & 1.0 & & 3 \\
\hline
\end{tabular}

Normal upper limit of temporal depression: 0.086 in upper field, 0.084 in lower field. Normal upper limit of pairs: three pairs with 2 dB or more sensitivity, two pairs with $3 \mathrm{~dB}$ or more sensitivity. Underlined bold number indicates abnormal. N indicates normal with Goldmann perimeter. A, abnormal. GH indicates growth hormone; NF, non-functioning; and PRL, prolactin.

1) showed increased temporal index with MRI grade (from grade 2 to 3 ) and underwent removal surgery. The other cases had no change of MRI grade.

\section{Discussion}

We determined new criteria for temporal hemianopia, vertical step and temporal depression. These criteria had high sensitivity, high specificity, and reproducible results. Some patients with pituitary tumor and no visual symptoms in the present study were found to have temporal hemianopia observed by automated perimetry, but not by Goldmann perimetry. These changes were thought to be initial alterations of temporal hemianopia due to chiasmal lesions. The visual field defects caused by chiasmal lesion have been estimated by Goldmann perimetry, but the determination of hemianopia was not sufficient with the use of that instrument alone. Anderson et al reported that patients with pituitary adenoma had few visual symptoms, ${ }^{6}$ as measured by Goldmann perimetry. Another study that used automated perimetry showed greater incidence of visual symptoms in patients with pituitary adenoma. ${ }^{7}$ There were some possible explanations for the superiority of automated perimetry. One was numeric values as sensitivity by automated perimetry. Vertical step or temporal depression observed by automated perimetry would increase the ability to detect visual disturbances in patients with pituitary adenoma. Mills pointed out at least three adjacent pairs were needed to find vertical step by automated perimetry for hemianopia. ${ }^{5}$ We obtained at least four pairs with $2 \mathrm{~dB}$ or more difference and three pairs with $3 \mathrm{~dB}$ or more difference from normal data for temporal hemianopia. Four pairs along the vertical median with $2 \mathrm{~dB}$ or more difference in sensitivity were easy to diagnose as an initial change of temporal hemianopia. The Humphrey field analyzer printed out pattern and total deviation for abnormal points. Abnormal points at some probability 
were shown on pattern and total deviation maps. We could identify abnormal areas on these deviation fields (Figure 5). Vertical step was not always found on these maps (Figure 6). Actual value was important for diagnosing hemianopia. Other explanations were thought to be due to statokinetic dissociation ${ }^{8}$ and testing procedure of automated perimetry. ${ }^{9}$ The Humphrey Field Analyzer threshold program (central 30-2) determines primary points in each visual field quadrant first. The primary points threshold is used for the initial value of the eight neighboring points surrounding the primary points and finally could affect the values in the entire quadrant. A lower value in the primary point may lead to lower values in the quadrant including the primary point.
Seven patients had $2 \mathrm{~dB}$ vertical step, but it was monoocular in six. Compression to the optic chiasm induced bilateral visual field changes, but monotemporal visual field defects could be present with chiasmal lesions. ${ }^{10}$ Initial temporal hemianopia might occur unilaterally, even as seen with automated perimetry. We chose only one line along the vertical meridian for vertical step. Temporal depression covered the other area. A temporal depression index was also useful to monitor initial change in temporal hemianopia. We could also use this index to follow up patients with asymptomatic temporal hemianopia. If this index increased, it would mean that compression would have progressed with enlargement of the tumor. Patients who underwent surgery all had a significantly

\section{Value (dB)

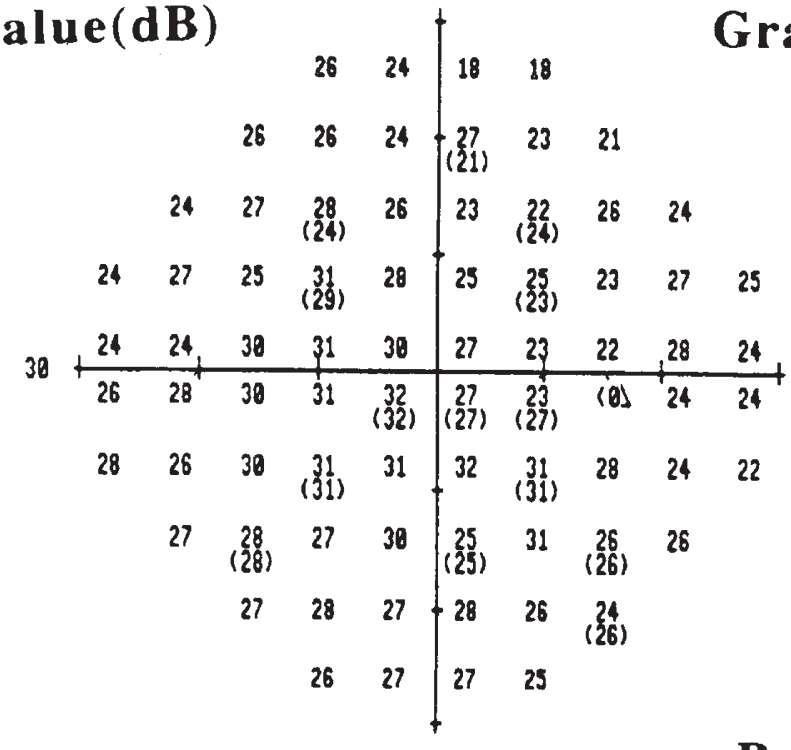

Total

Deviation

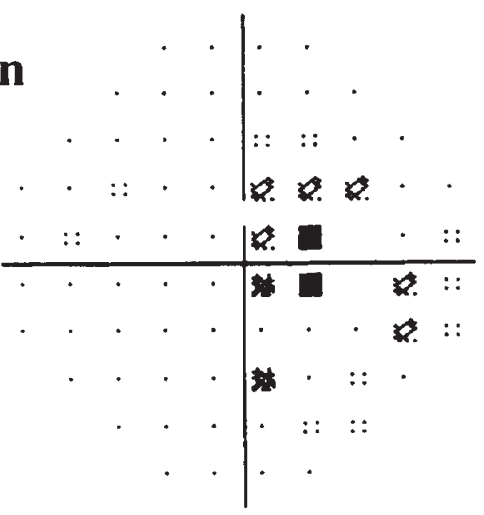

\section{Pattern}

\section{Deviation}

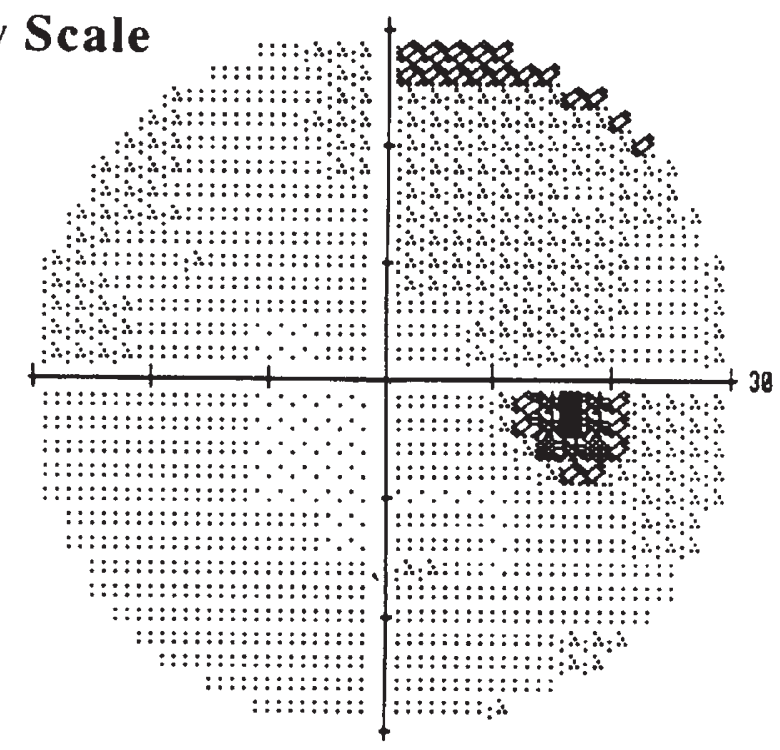

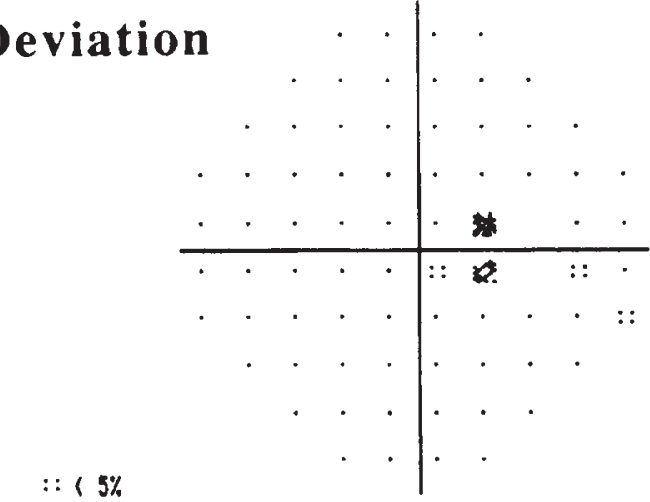

2. $12 \%$

* $11 \%$

Figure 5 Patient 4 (36-year-old woman with prolactinoma and MRI grade 1) shows single field analysis. Right field has four adjacent pairs with $3 \mathrm{~dB}$ or more sensitivity and four adjacent abnormal points in total deviation. 


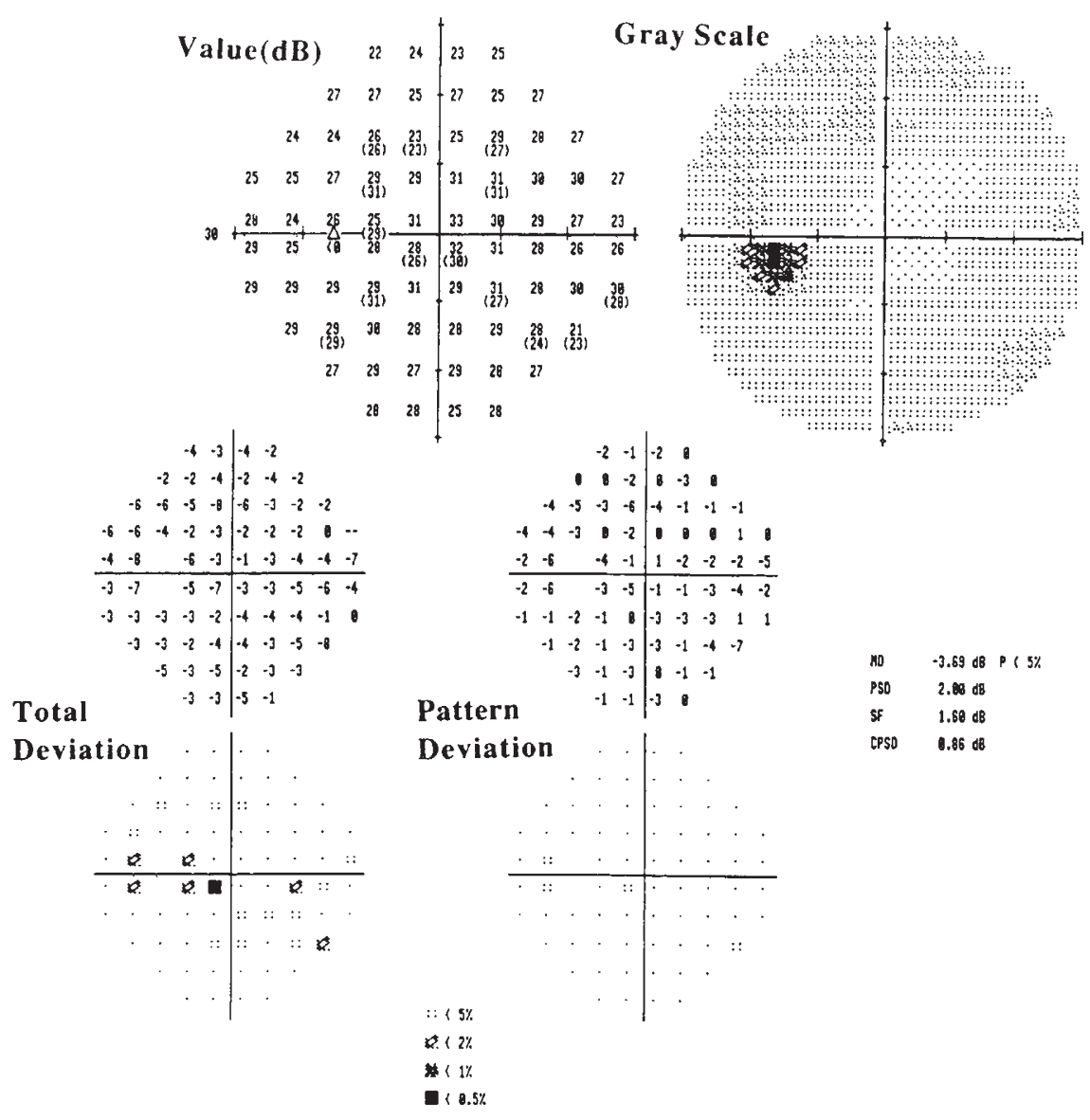

Figure 6 Patient 11 (22-year-old woman and MRI grade 2) shows single field analysis. Left field has five adjacent pairs with $2 \mathrm{~dB}$ or more sensitivity and only two abnormal points along with the midline in total deviation.

increased index. This index could be a criterion for surgical indication.

We believed that what produced different indexes between patients with MRI grade 3 and grade 3+ was duration of compression. Symptomatic patients (MRI grade $3+$ ) who had hemianopia observed by Goldmann perimetry had a greater index. It was possible that the optic chiasm in these patients was compressed by the tumor longer than in asymptomatic patients (MRI grade 3) who had no hemianopia found on Goldmann perimetry. Longer compression to the chiasm induced more functional loss by automated perimetry.

Our new MRI grading system was useful for identifying patients with initial field loss without symptoms. Visual field defects with Goldmann perimetry appeared as the tumor enlarged. ${ }^{11} \mathrm{We}$ focused on compression to and elevation of the chiasm. Grade 3 compression could induce hemianopia detected by automated perimetry, but not always by Goldmann perimetry. Grade 4 compression resulted in visual field loss detected by both Goldmann perimetry and automated perimetry. Grade 3 and 4 compressions also distorted the optic nerve and tract equally, but induced no functional loss in such pathways. Direct compression could produce hemianopia, but not distortion. Even two cases of grade 1 compression produce visual field loss. Only contact with the optic chiasm hardly caused field loss, although the tumor may have compressed the optic chiasm previously and then decreased in size. Pituitary adenoma occasionally has decreased in size with time. ${ }^{12}$ Both the findings of MRI and automated perimetry could be helpful in the management of asymptomatic pituitary tumor.

\section{Acknowledgements}

Supported in part by an Aid of Scientific Research, Japan (No. 13671822), Ministry of Education, Culture, Sports, Science and Technology.

\section{References}

1 Elkington SG. Pituitary adenoma: pre-operative symptomatology in series of 260 patients. $\mathrm{Br}$ J Ophthalmol 1968; 52: 322-328. 
2 Grochowicki M, Vighetto A, Berquet S, Khalfallah Y, Sassolas G. Pituitary adenoma: automatic static perimetry and Goldmann perimetry. A comparative study of 345 visual field charts. Br J Ophthalmol 1991; 75: 219-221.

3 Beck RW, Bergstrom TJ, Lichter PR. A clinical comparison of visual field testing with a new automated perimeter, the Humphrey field analyzer, and the Goldmann perimeter. Ophthalmology 1985; 92: 77-82.

4 Lee AG, Miller NR. Neuro-ophthalmological findings in pituitary adenomas. In: Landort AM, Vance ML, Reilly PL (eds). Pituitary Adenomas. Churchill Livingstone: New York, 1996, pp 157-175.

5 Mills RP. Automated perimetry in neuro-ophthalmology. Int Ophthalmol Clin 1991; 31: 51-70.

6 Anderson D, Faber P, Marcovitz S, Hardy J, Lorenzetti D. Pituitary tumors and the ophthalmologist. Ophthalmology 1983; 90: 1265-1270.

7 Bynke H. Pituitary adenomas with ocular manifestations.
Incidence of cases and clinical findings 1946-1984. Neuroophthalmology 1986; 6: 303-311.

8 Safran AB, Glaser JS. Statokinetic dissociation in lesions of the anterior visual pathways. A reappraisal of the Riddoch phenomenon. Arch Ophthalmol 1980; 98: 291-295.

9 Wall M, Johnson CA, Kutzko KE, Nguyen K, Brito C, Keltner JL. Long- and short-term variability of automated perimetry results in patients with optic neuritis and healthy subjects. Arch Ophthalmol 1998; 116: 53-61.

10 Hershenfeld SA, Sharpe JA. Monocular temporal hemianopia. Br J Ophthalmol 1993; 77: 424-427.

11 Ikeda H, Yoshimoto T. Visual disturbances in patients with pituitary adenoma. Acta Neurol Scand 1995; 92: 157160 .

12 Igarashi T, Saeki N, Yamaura A. Long-term magnetic resonance imaging follow-up of asymptomatic sellar tumors. Neurol Med Chir (Tokyo) 1999; 39: 592-599. 